\title{
La investigación acción educativa en educación afectivosexual: una metodología para el cambio social
}

\author{
Mar Venegas Medina \\ Universitad de Granada \\ mariter@ugr.es
}

Recibido: 01.10.2009

Aceptado: 13.10 .2010

\section{INTRODUCCION}

Dice la metodóloga británica Berg (2001: 71) que «el mundo es un laboratorio de investigación», y ésa es la premisa de la que parte este artículo, y mi propio trabajo como socióloga: de entender que cualquier espacio de nuestra vida cotidiana merece ser reflexionado como espacio para la investigación social. Sin embargo, yo quisiera dar un paso más. Merece ser investigado siempre que, seguidamente, sea considerado desde la perspectiva de la ciencia social aplicada, como espacio para promover, mediante la acción investigadora, el cambio social; especialmente cuando ese espacio está enmarcado en el contexto de la educación formal. En estas coordenadas, casi como una declaración de principios, me sitúo al plantear este artículo, donde recojo todo el proceso metodológico de mi investigación de tesis doctoral sobre educación afectivosexual en secundaria, en el que he entendido la práctica investigadora y la educativa como prácticas sociales complementarias en la tarea de contribuir, desde la Educación Secundaria Obligatoria, a la transformación de las desigualitarias estructuras sociales de género y sexualidad hacia un modelo basado en la igualdad. Para ello, la socialización igualitaria de los sujetos adolescentes resulta fundamental; educar en igualdad es invertir en la transformación intergeneracional de la estructura social. El proceso subyacente a todo ello es el trabajo metodológico del que pretendo dar cuenta en este artículo, donde la práctica sociológica investigadora, como práctica educadora simultáneamente, adquiere un importante potencial transformador de la desigualdad sexual y de género.

Si la sociología nació, entre otras cosas, como disciplina de conocimiento del cambio social, para quienes nos situamos en la teoría crítica como espacio de conocimiento de nuestra sociedad, la sociología puede ser, en su dimensión de ciencia social aplicada, un instrumento magnífico que contribuya al cambio so- 
cial, especialmente en temas tan sociales como la igualdad sexual y de género, la lucha contra la violencia de género, o la vivencia de la sexualidad y la afectividad en la adolescencia sin tabúes, sin represiones, como parte integral del ser humano. Y todo ello sin dejar de ser una disciplina académica sistemática, manteniendo en todo momento la calidad científica también en la investigación social aplicada. En las siguientes páginas quisiera, pues, describir esta apasionante aventura de la investigación social, poniendo el énfasis en lo que la metodología de análisis sociológico puede hacer para contribuir también al cambio social, una vocación feminista por excelencia, tal como afirma Butler (2001) al definir el feminismo como práctica social transformadora.

El objetivo principal de esta investigación es arrojar luz sobre la educación afectivosexual desde una aproximación sociológica, puesto que nada se había investigado en España sobre este tema desde la sociología. Así pues, utilizando el término de Berg (2001), ha sido una «investigación-antes-de-la-teoría (proceso inductivo)», si bien, el punto de partida estuvo en la revisión de los materiales sobre educación sexual y afectivosexual publicados en Andalucía, España y otros países de referencia como Reino Unido y Estados Unidos ${ }^{1}$.

En el diseño del esquema metodológico de la investigación, sigo la propuesta de Mason (1996) sobre la esencia de la investigación:

- La naturaleza del fenómeno u ontología del proyecto: valores y normas, localizadas en el nivel simbólico de los «regímenes de género»² (Connell 1987, 2002); y prácticas, que tienen lugar en la estructura material de los diferentes regímenes de género, y están orientadas por esos valores y normas. Para investigarlo, Mason (1996) propone, entre otras, la entrevista como técnica muy adecuada para la recogida de datos de esta naturaleza.

- El conocimiento/evidencia o teoría del conocimiento: que, en esta investigación, deriva en lo que he llamado 'teoría postestructuralista de la práctica', como revisión postestructuralista de la teoría de la práctica ${ }^{3}$.

${ }^{1}$ Esta investigación se ha desarrollado principalmente en Andalucía por ser una de las comunidades españolas de mayor desarrollo institucional de la coeducación, como marco de fondo para la educación afectivosexual basada en la igualdad sexual y de género; además, es la comunidad donde desarrollo mi trabajo académico. La elección de RU y EEUU como países de referencia se debe al desarrollo que en los mismos tiene la educación de las relaciones afectivosexuales en el currículum formal, y la investigación social en torno a ello, prueba de lo cual es la existencia de sus respectivos organismos oficiales, SEF (Sex Education Forum, integrado en el National Children Bureau) y SIECUS (Sexuality Information and Education Council of the United States).

2 Sigo aquí el concepto de Robert William Connell $(1987,2002)$ de «régimen de género» como modelo históricamente construido de relaciones entre mujeres y hombres y las definiciones de masculinidad y feminidad. Más adelante volveré a esta cuestión más detenidamente.

${ }^{3}$ Puesto que este artículo versa sobre la metodología de la investigación, no me detendré a entrar en cuestiones epistemológicas que, sin embargo, permean constantemente las decisiones metodológicas de la investigación, por lo que han sido consideradas durante todo el proceso investigador, aunque no sea éste el espacio destinado a hablar de ello. Para profundizar en esta cuestión, ver Venegas (2009a).

EMPIRIA. Revista de Metodología de Ciencias Sociales. N. ${ }^{\circ}$ 21, enero-junio, 2011, pp. 39-61. ISSN: 1139-5737 
- El tema de investigación: que lo constituye la política de las relaciones afectivosexuales (política afectivosexual), la cual se desglosa en cuatro subtemas o estructuras sociales, a saber: género, sexualidad, afectividad y cuerpo.

- Las preguntas de investigación: que se derivan de todo el trabajo teóricoepistemológico de definición y acotación del objeto de estudio de mi investigación, y que, sin embargo, exceden el objetivo y los contenidos de este artículo, por lo que no me detendré a explicar la revisión bibliográfica del trabajo hasta llegar a formular las preguntas de investigación, aunque sí es pertinente en este artículo plantear la pregunta general de investigación: ¿cómo tienen lugar, a nivel microsociológico, los procesos de socialización y subjetivación, y a nivel macrosociológico, de reproducción y cambio, en torno a las cuatro estructuras integrantes de la política afectivosexual -género, sexualidad, afectividad y cuerpo-, en las chicas y chicos de los dos colegios -el de clase obrera y el de clase media- de la investigación?.

- El propósito de la investigación o contexto sociopolítico de la práctica investigadora: en este caso la coeducación, en que la educación afectivosexual emerge como una de las cuestiones fundamentales y pendientes de las políticas educativas para la intervención educativa en favor de la igualdad sexual y de género, con el objetivo último de erradicar la violencia de género, por lo que la política afectivosexual es el contexto socioestructural por excelencia para entender la lógica de los procesos sociales subyacentes, a fin de arrojar luz sobre el fenómeno de la educación afectivosexual, con dos motivos: sociopolíticamente, para contribuir a la elaboración de una política educativa adecuada; y, epistemológicamente, para vincular la producción de teoría con la práctica investigadora y educativa, para hacer de la investigación acción educativa una práctica de cambio social hacia la igualdad.

Esta investigación surge ante la constatación de una evidencia y la necesidad de ser investigada sociológicamente. Primero, concretamente en el colegio de clase obrera de la ciudad de Granada donde se ha desarrollado el trabajo de campo, observamos complejos fenómenos sociales relacionados con la educación afectivosexual como patriarcado, sexismo, violencia (física y simbólica), carencias afectivas, embarazos adolescentes; entendimos, entonces, la necesidad de investigarlos desde la perspectiva comparada de clase, género y etnia (Venegas y Echeverría 2005/06, 2006; Venegas 2007a, 2007b), lo que dirigió la metodología de la investigación. El primer año fue un Estudio Piloto para sentar las bases del trabajo de campo posterior, mediante un curso de educación afectivosexual con un grupo de $3^{\circ}$ ESO, Letras, de ese colegio, durante el curso 2004/05 (Venegas y Echeverría 2005/06). El curso siguiente, empezamos el trabajo de campo como tal, con ese mismo grupo, que pasaba a ser $4^{\circ}$ ESO Letras. El tercer año, replicamos la investigación con un grupo $4^{\circ}$ ESO Letras en un colegio de clase media de la misma ciudad.

EMPIRIA. Revista de Metodología de Ciencias Sociales. N. ${ }^{\circ}$ 21, enero-junio, 2011, pp. 39-61. ISSN: $1139-5737$ 


\section{LOS OBJETIVOS DE LA INVESTIGACIÓN}

Dada la ausencia de investigación sociológica sobre educación afectivosexual en el contexto español, este trabajo fue concebido como estudio exploratorio sobre ello, buscando, como señala Elliot (1993), no tanto elaborar leyes generales o conclusiones generalizables, sino las dinámicas subyacentes a los estudios de casos para aprender de ellos. De ahí la importancia de tomar como referencia los casos británico o estadounidense, donde hay amplia tradición en la investigación social sobre este tema.

Así, la investigación parte con el objetivo general de analizar los valores, normas y prácticas sobre género, sexualidad, afectividad y cuerpo, en los regímenes de género que son barrio, familia, escuela, grupo de amistad y pareja, para estudiar los procesos de socialización y subjetivación que tienen lugar en torno a ello en la adolescencia, entendidos como procesos microsociológicos que se corresponden con procesos macrosociológicos de reproducción y cambio respectivamente, y teniendo en cuenta las perspectivas comparativas de clase social, género y etnia. Dar cobertura a este objetivo significa aproximarse exploratoriamente a la política afectivosexual en la adolescencia. En este objetivo están contenidas las dimensiones epistemológicas y ontológicas de la investigación, que señalaba antes al referirme a la «esencia de la investigación» siguiendo a Mason (1996), y que han guiado todo el proceso, por lo que considero importante abordarlas con más detalle en un artículo sobre metodología de investigación social como éste.

Lo primero es definir las unidades de análisis y la dimensión ontológica de la investigación (Mason 1996). Para dar cobertura al objetivo por excelencia de esta investigación, sobre los procesos de socialización (Connell 1987, 2002) y subjetivación (Butler 2001) relativos a la política afectivosexual en la adolescencia, es necesario analizar los valores y normas -como unidades de análisisque mueven las prácticas de las chicas y chicos de la investigación. Estas cuestiones sitúan la investigación en el nivel microsociológico de análisis, incompleto si no se remite, simultáneamente, al nivel macrosociológico constituido por las estructuras discursivas y materiales en que quedan contextualizados esos valores, normas y prácticas. La conexión epistemológica entre valores y normas, prácticas y estructuras sociales ha sido ampliamente desarrollada por la teoría de la práctica (Connell 1987, 2002; Holland y Eisenhart 1990; Skeggs 1997; Bourdieu 2005; Kemmis 2007) y es referente fundamental en mi trabajo.

El siguiente paso es localizar esas unidades de análisis en espacios sociales empíricamente abordables y relevantes para esta investigación. Para ello, utilizo el concepto de regímenes de género ${ }^{4}$ (Connell, 1987). En concreto, aquéllos donde la interacción es directa o inmediata, a saber: barrio, familia, escuela, grupo

\footnotetext{
${ }^{4}$ Ver definición en nota a pie de página número 2.
}

EMPIRIA. Revista de Metodología de Ciencias Sociales. N. ${ }^{\circ}$ 21, enero-junio, 2011, pp. 39-61. ISSN: 1139-5737 
de amistad ${ }^{5}$ pareja $^{6}$, lo que supone el nivel mesosociológico de análisis de la investigación. De este modo, es posible analizar los diferentes niveles sociológicos de la política afectivosexual y las relaciones entre ellos. Asimismo, he considerado imprescindible adoptar las perspectivas comparativas de clase social, género y etnia, especialmente cuando el tema se aborda desde la teoría social feminista y crítica.

Para poder llevar a cabo este estudio exploratorio, fue necesario diseñar una metodología que hiciera posible partir de lo que se había estudiado, la educación afectivosexual, para llevarlo al terreno de la investigación sociológica. Este proceso queda registrado en el giro terminológico y epistemológico que supone el paso de la educación a la política afectivosexual, donde ésta no se refiere tanto al hecho de educar las relaciones afectivosexuales, cuanto a la fundamentación -e investigación- del espacio socioestructural en que tienen lugar las relaciones afectivosexuales, como un tipo concreto de relación social (Venegas 2009a). Si la educación afectivosexual es objeto de estudio de pedagogía, psicopedagogía y didáctica, la política afectivosexual es objeto de estudio sociológico, al definirla como una red de «relaciones sociales que implican poder o autoridad $\gg^{7}$ en torno a las cuatro estructuras sociales que la componen: género, sexualidad, afectividad y cuerpo. La determinación de estas cuatro estructuras, lejos de ser arbitraria, se deriva del trabajo que he realizado para categorizar los temas comprendidos en los materiales revisados sobre educación afectivosexual, llegando a la conclusión de que su agrupación se corresponde con estas cuatro áreas (Venegas 2009b) .

Ambas dimensiones del trabajo -educación y política afectivosexual- quedan perfectamente equilibradas, así, en una línea de continuidad que empieza por la investigación de la educación afectivosexual, en el aula, con cada uno de los dos grupos-clase, siguiendo una serie de objetivos que han estructurado cronológicamente las fases del trabajo de campo. En primer lugar, revisar los programas y materiales sobre educación afectivosexual; principalmente los editados en Andalucía, pero también en Granada, ciudad donde se desarrolla la investigación, el resto de España y otros países de referencia como Reino Unido o Estados Unidos. Con esta revisión, el objetivo es doble: 1) conocer sus valores, principios y propuestas; y 2) detectar los temas y cuestiones relevantes en educación afectivosexual para categorizarlos en áreas temáticas. De ello se deriva el tercer ob-

\footnotetext{
${ }^{5}$ Lo que en sociología se denomina grupo de pares o grupo de iguales.

6 Por pareja entiendo aquí aquella forma de relación afectivosexual constituida por dos miembros que, en este caso, son adolescentes y heterosexuales. No ha sido empíricamente viable la investigación de parejas adolescentes homosexuales, dada la no identificación de las mismas en ella, si bien, estaban comprendidas a priori como forma potencial de relación adolescente, de la que hemos hablado además en las sesiones de clase.

7 Así se define el término politics en su $4^{\circ}$ acepción, «social relations involving authority or power», según el Diccionario WordReference, en http://www.wordreference.com/definition/politics (última consulta 14.10.2009). Traducción del original en inglés al español mía.

${ }^{8}$ Más adelante (apartado 4.3.; tabla 2) volveré a esta cuestión.
} 
jetivo, marcado por la metodología constructivista predominante en estos materiales revisados: elaborar el programa del curso de educación afectivosexual impartido en cada grupo-clase ${ }^{9}$. Al respecto, como cuarto objetivo, estimular la participación de las y los estudiantes en clase, para lo cual utilizo técnicas basadas en la dinamización de grupos, bien propuestas en los materiales revisados, bien diseñadas por mí. Con todo ello, el objetivo es investigar y educar simultáneamente en el aula las relaciones afectivosexuales adolescentes.

La fase de trabajo en el aula fue seguida por la investigación de la política afectivosexual, sistematizando el objetivo general arriba señalado y para el que la entrevista fue elegida como la técnica de producción de datos más adecuada, tal como explicaré más adelante. Esta fase de la investigación comprende dos niveles sociológicos de contextualización. Por un lado, el contexto socioestructural más inmediato, el barrio, para investigar comparativamente la dimensiones étnica y de clase (McRobbie 1978; Holland y Eisenhart 1990; Skeggs 1997; Bourdieu 1999; Willis 2003; Fernández, Venegas y Olmedo 2004), de manera que he realizado el trabajo de campo en dos colegios, uno situado en un barrio tradicionalmente de clase obrera de la ciudad de Granada, y otro en pleno centro de la ciudad, de clase media. Por otro lado, mediante el concepto de 'régimen de género' de Connell $(1987,2002)$, antes mencionado ${ }^{10}$, para analizar los valores, normas y prácticas relativos a género, sexualidad, afectividad y cuerpo, a fin de investigar los procesos de socialización y subjetivación implicados en la política afectivosexual. Siguiendo a este autor, barrio, familia, escuela, grupo de amistad y pareja son regímenes de género, en concreto, los que he considerado relevantes para mi investigación por su incidencia en la política afectivosexual adolescente. Su análisis hace posible investigar las dimensiones estructurales material y simbólica para conocer la estructura de las relaciones que tienen lugar en cada uno de esos regímenes de género.

De cada colegio, he seleccionado un grupo-clase con el que trabajar en profundidad las dos dimensiones, educación y política afectivosexual. En concreto, he optado por un grupo de $4^{\circ}$ de Educación Secundaria Obligatoria, por entender que se trata de un momento representativo de los cambios sociales, psicológicos y fisiológicos de la adolescencia en lo relativo a la política afectivosexual. Por la propia naturaleza de la investigación, las y los sujetos de la misma han sido todas las chicas y chicos de los dos grupos-clase. La siguiente tabla recoge su distribución:

Tabla 1. Distribución de sujetos de la investigación

\begin{tabular}{|l|c|c|c|}
\hline & Clase obrera & Clase media & Total \\
\hline Chicas & 7 & 5 & $\mathbf{1 2}$ \\
\hline Chicos & 7 & $8 / 23$ & $\mathbf{3 0}$ \\
\hline Total & $\mathbf{1 4}$ & $\mathbf{2 8}$ & $\mathbf{4 2}$ \\
\hline
\end{tabular}

\footnotetext{
9 Ver tabla 2.

10 Ver nota 2.
}

EMPIRIA. Revista de Metodología de Ciencias Sociales. N. ${ }^{\circ}$ 21, enero-junio, 2011, pp. 39-61 ISSN: 1139-5737 
De este modo, he trabajado con un total de 42 sujetos, 12 chicas y 30 chicos. En el grupo-clase del colegio de clase obrera había 7 chicas y 7 chicos. En el de clase media, había una sobrerrepresentación de chicos, 23, y sólo 5 chicas, por lo que, en la fase de análisis de las entrevistas, hice una primera revisión para seleccionar las más representativas del total de chicos de este grupo, siendo finalmente 8 las analizadas. Sus edades están comprendidas entre los 15 y los 18 años, siendo la más frecuente 16 años.

Parecía adecuado, entonces, considerar cada grupo-clase como un estudio de caso, siguiendo la definición de Berg (2001: 225), para quien se trata de una:

Colección sistemática de información suficiente sobre una (...) situación social particular (...) que permite a quien investiga entender efectivamente cómo opera o funciona (...). No es verdaderamente una técnica de recogida de datos, sino una metodología de aproximación que incorpora una serie de medidas de recogida de datos.

Efectivamente, esta compleja planificación metodológica generaba las condiciones necesarias para producir datos con los que investigar dos situaciones sociales particulares o concretas sobre la política afectivosexual adolescente susceptibles de ser comparadas en términos de clase, género y etnia. Asimismo, como metodología aproximativa, integra una serie de técnicas de recogida de datos que iré detallando a continuación. Cada sesión de clase, como espacio de observación participante, producía datos relativos al tema trabajado, e iba generado las condiciones adecuadas para la realización posterior de las entrevistas en profundidad, sentando las bases de una complicidad que haría posible la riqueza e intimidad de las entrevistas sobre temas de difícil acceso para la investigación social como los tratados en esta investigación.

\section{UNA METODOLOGÍA PARA EL CAMBIO SOCIAL: LA INVESTIGACIÓN ACCIÓN EDUCATIVA EN EDUCACIÓN AFECTIVOSEXUAL}

Por su naturaleza y objetivos, esta investigación ha entrañado continuas reflexiones metodológicas al hilo del trabajo empírico y sus implicaciones teóricas y sociopolíticas. En tanto que educativa, la acción investigadora ha sido de compromiso científico y social, lo que me llevaba a repensar constantemente la dimensión ética de la investigación. Y ello aún cuando tradicionalmente la sociología ha basado su cientificidad en el hecho de prescindir de este tipo de cuestiones. Pese a todo, esta investigación adquiere un compromiso ético desde la investigación acción educativa como metodología participativa y crítica orientada a contribuir al cambio social hacia la igualdad sexual y de género. 


\section{Criterios de cientificidad}

El compromiso ético y social en la investigación es, a mi entender, totalmente compatible con los criterios de cientificidad, la sistematización del trabajo y el esfuerzo teórico y metodológico por cubrir el abanico de posibles referentes. En este sentido, sigo a Mason (1996), quien insiste en la necesidad de cumplir con tres requisitos fundamentales de cientificidad, a saber: validez, esto es, medir lo que se pretende medir; fiabilidad, o adecuación y precisión de los métodos y la técnicas empleados; y generalizabilidad, o alcance de las conclusiones obtenidas, que ha de supeditarse, como señala Elliot (1993), al relativo potencial generalizador de las conclusiones microespaciales que pueden extraerse de los trabajos de investigación acción educativa, ya que suelen basarse en estudios de casos.

\section{La investigación acción educativa}

La elección de la investigación acción educativa como la metodología apropiada para este trabajo se deriva de la revisión de los materiales sobre educación sexual y/o afectivosexual existentes (Urruzola 1991; Barragán 1999; Gorrotxategui y de Haro 1999; Gómez 2004). Dadas la temática y el planteamiento de esta investigación, resultaba insuficiente utilizar una metodología cualitativa estática, pasiva, que fuera al campo a recoger datos. A ello se suma el carácter exploratorio de la investigación. De ahí esta apuesta por la reflexión sobre la investigación desde una práctica investigadora que educa, al mismo tiempo, y cuyos resultados han de tener una dimensión claramente aplicada, en tanto que han de ponerse al servicio de las políticas educativas no sólo para tomar decisiones políticas, sino también curriculares. Pero esta orientación práctica no exime de un trabajo epistemológico y ontológico serio en la investigación social. Las bondades aplicadas de la investigación se derivan de su calidad teórica.

Es Stenhouse (1987) el primero en reflexionar sobre la práctica del currículum. Más tarde, Elliott $(1993,1994)$ propone la promoción del cambio educativo desde una concepción de la investigación acción en educación que podemos considerar como de inspiración colaboradora entre quienes investigan y quienes ejercen la práctica docente. Posteriormente, Carr (1997) pone el énfasis en una investigación acción participativa e inspirada en la teoría de la acción comunicativa de Habermas, dentro de la teoría crítica de la enseñanza. La propuesta de mi trabajo está más próxima a la investigación acción colaborativa de Elliott (1993, 1994), junto al referente fundamental de Kemmis (2007), quien entiende la investigación acción como una práctica para el cambio de prácticas, pues comprende tres dimensiones de cambio interrelacionadas: las prácticas ('doings'), la comprensión de esas prácticas ('sayings') y las condiciones en que las prácticas tiene lugar ('relatings'), o sea,

EMPIRIA. Revista de Metodología de Ciencias Sociales. N. ${ }^{\circ}$ 21, enero-junio, 2011, pp. 39-61. ISSN: 1139-5737 
trabajo, lengua y poder. Siguiendo la teoría de la práctica, señala que «práctica, prácticos/as, y condiciones de la práctica son transformadas tanto como reproducidas de una ocasión a otra» ${ }^{11}$ (Kemmis 2007: 5), lo que enlaza con los objetivos epistemológicos de esta investigación. Kemmis afirma que, para que el cambio se consolide, necesita de la coherencia de la nueva interrelación, a pesar de que la contradicción y el conflicto puedan formar parte de ello.

\section{La investigación acción en educación sexual}

Mi investigación parte de la consideración de la educación como herramienta de cambio social. De ahí mi interés por hacer de esa experiencia educativa un trabajo sociológico de reflexión empírica y teórica. Todas las experiencias de campo han sido, así, un proceso continuo de reflexión teórica y autorreflexión de las prácticas educativas e investigadoras. Hay una ruptura con el discurso cientificista, empirista, objetivista del positivismo, porque me interesan las personas, sus afectos, sus experiencias, sus relaciones. Y ello implica respeto a los criterios de cientificidad. Una investigación sobre afectos no podía hacerse sin afectos. Lo primero ha sido tratar a las y los sujetos como lo que son: personas, sujetos agentes, protagonistas de los procesos que estábamos viviendo en el aula.

En los materiales revisados sobre educación afectivosexual (Urruzola 1991; Barragán 1999; Gorrotxategui y de Haro 1999; Gómez 2004), hay una apuesta clara por la metodología de la investigación acción educativa por las aportaciones de la misma a este objeto de estudio, de manera que podemos extraer algunas conclusiones metodológicas fundamentales:

1. La adopción de un enfoque constructivista en el proceso de enseñanzaaprendizaje, para promover el aprendizaje mediante la construcción grupal de las nociones sexuales, siempre desde la perspectiva de género (Barragán 1999), y estimular una transformación de las teorías personales del alumnado, respetando el proceso natural de construcción de las mismas (Gorrotxategui y de Haro 1999). Barragán (1999) propone elaborar el currículo a partir de las demandas del grupo-clase como resultado de la investigación en la acción y para introducir mejoras sucesivas, incluyendo también temas fundamentales que no han aparecido a demanda del estudiantado.

2. Los objetivos generales de la educación sexual en educación secundaria dan forma metodológica y curricular a la investigación acción educativa en el aula (Barragán 1999). Podemos sintetizarlos como sigue: construir un conocimiento sexual crítico con la sexualidad y el género para potenciar la capacidad de transformación; igualmente, potenciar la reflexión y el análisis sobre la cons-

${ }^{11}$ Traducción del original en inglés al español mía. 
trucción social de la sexualidad ${ }^{12}$, o sea, por qué nuestra cultura «regula» el comportamiento sexual en unos términos concretos a partir de lo normal y anormal; finalmente, eliminar estereotipos y eufemismos sobre género, sexualidad, afectividad y cuerpo, como proceso previo a la promoción de la igualdad desde la educación afectivosexual.

3. La metodología constructivista implica también la evaluación de los cambios promovidos por la educación afectivosexual desde la investigación acción educativa, utilizando una metodología de evaluación cualitativa ${ }^{13}$ (Barragán 1999).

4. Se enfatizan la necesidad de utilizar un lenguaje no impositivo, favoreciendo la argumentación, el debate y las reflexiones críticas en el aula (Barragán 1999; Gorrotxategui y de Haro 1999).

Todas estas premisas metodológicas han guiado mi trabajo, especialmente en lo relativo a la investigación de la educación afectivosexual que, como decía más arriba, se prolonga y completa con la investigación de la política afectivosexual, para lo que ha sido necesario diseñar un instrumento que permitiera dar continuidad al trabajo cualitativo de "colección sistemática de información suficiente sobre una (...) situación social particular» tal como vimos que decía Berg (2001: 225) al referirse a los estudios de casos. Ese instrumento ha sido la entrevista, a la que dedico el siguiente apartado.

\section{UNA METODOLOGÍA CUALITATIVA PARA DESCRIBIR, INTERPRETAR, REFLEXIONAR}

Investigar, especialmente en ciencias sociales, tiene una naturaleza política y otra moral fundamentales. En este sentido, sigo la apuesta de Mason (1996) por una metodología cualitativa, activa, donde se vinculen las implicaciones intelectuales, prácticas, éticas y políticas. Mason sitúa esta metodología en la tradición sociológica interpretativa, donde 'cualitativa' se refiere a una posición filosófica que es interpretativa, centrada en significados, interpretaciones, prácticas, discursos, procesos y construcciones. Para proceder adecuadamente, Mason señala una serie de requisitos básicos para la metodología cualitativa, que ha de ser sistemática y rigurosa; estratégica pero flexible y contextual; practicar la reflexividad activa, para pensar en todo momento el rol de quien investiga; producir explicaciones sociales que tengan una resonancia amplia; ser conducida como una práctica ética en relación a su contexto político. Además, es un proceso cíclico, por lo que implica movimientos continuos de ida y vuelta.

${ }^{12}$ La literatura sobre sexualidad desde las ciencias sociales ha sentado las bases teóricas para esta propuesta que se lleva así al aula, a la educación sexual, lo que evidencia el vínculo entre teoría sociológica y desarrollo curricular de esa teoría: planteamiento teórico y práctica educativa se unen.

${ }^{13}$ La fase de las entrevistas que comprende la metodología de esta investigación está pensada, de alguna forma, como evaluación del trabajo realizado previamente en clase.

EMPIRIA. Revista de Metodología de Ciencias Sociales. N. ${ }^{\circ}$ 21, enero-junio, 2011, pp. 39-61. ISSN: 1139-5737 


\section{La técnica de la entrevista en profundidad}

Si recordamos los objetivos, derivados de la naturaleza ontológica y epistemológica de esta investigación, es fácil comprobar que las propuestas metodológicas que vengo planteando se van amoldando a ello. De ahí la entrevista como técnica más idónea para producir los datos sociológicos de la investigación que permiten acceder al mundo simbólico de cada sujeto, como lo que origina sus prácticas. Por ello, he considerado la entrevista como la técnica más importante de producción de datos en la investigación de la política afectivosexual dentro de esta metodología cualitativa crítica.

Así, Jones (1985: 46) rompe con conductismo y determinismo cultural en investigación social y defiende la entrevista porque permite acercarse a:

La persona, quien construye significados y significaciones sobre su realidad. (...) Para entender por qué las personas actúan de la manera que lo hacen, necesitamos entender los significados y significaciones que dan a sus acciones ${ }^{14}$.

Por su parte, Taylor y Bogdan (1986: 101) definen la entrevista cualitativa en profundidad en términos interaccionales, como:

Reiterados encuentros cara a cara entre el investigador y los informantes, encuentros éstos dirigidos hacia la comprensión de las perspectivas que tienen los informantes respecto de sus vidas, experiencias o situaciones, tal como las expresan con sus propias palabras.

Siguen, por tanto, «el modelo de una conversación entre iguales» (Ibíd.). En este sentido, en mi investigación, sólo gracias al trabajo previo en el aula fue posible que las entrevistas produjesen datos no sólo abundantes sino muy ricos. Taylor y Bogdan consideran las entrevistas en profundidad especialmente adecuadas para algunas situaciones, como cuando «los escenarios o las personas no son accesibles de otro modo (...) la investigación depende de una amplia gama de escenarios o personas» (Taylor y Bogdan 1986: 105), y «el investigador quiere esclarecer experiencia humana subjetiva» (p.106), como ocurre en mi investigación, donde se busca la aproximación subjetiva (emic) de cada sujeto a su propia experiencia en diferentes regímenes de género inaccesibles mediante otra técnica. Pero los autores advierten que:

En tanto forma de conversación, las entrevistas son susceptibles de producir las mismas falsificaciones, engaños, exageraciones y distorsiones que caracterizan el intercambio verbal entre cualquier tipo de personas (Ibíd.).

\footnotetext{
${ }^{14}$ Traducción del original en inglés al español mía.
} 
Berg (2001) defiende la entrevista como mejor técnica para conocer las percepciones de las personas, sus comprensiones, o el apego al significado de ciertos fenómenos. Según su clasificación, el tipo de entrevista de esta investigación sería la estandarizada, con un esquema formalmente estructurado que permite comparar las respuestas entre las y los sujetos entrevistados; para lo que parte del supuesto de que todas las preguntas van a ser significativas y entendidas del mismo modo por todas las personas entrevistadas; y asume que los pensamientos y las acciones de las personas están íntimamente relacionados. Es focalizada porque utiliza un lenguaje cercano a las personas entrevistadas, como proponen también Barragán (1999) y Gorrotxategui y De Haro (1999).

Como vimos, Mason (1996) destaca que, en investigación cualitativa, la entrevista ha de considerar las dimensiones ontológica y epistemológica de la investigación, identificando «temas interpretativos» (Mason 1996: 40). Quien investiga ha de verse como una persona activa y reflexiva en el proceso de «generación de datos», término que prefiere al de «recogida de datos» (Ibíd.). Y enfatiza la dimensión ética de la entrevista, referida a la confidencialidad y el anonimato, las relaciones de poder y el consentimiento informado.

En un sentido similar al de Taylor y Bogdan (1986), Bourdieu (1991) considera que la entrevista crea una situación de comunicación que podemos entender como espacio de interacción entre quien investiga y quien se investiga, esto es, una relación social. Por tanto, la diferencia entre la ciencia positivista y «un uso reflexivo de las conquistas de la ciencia social» (Bourdieu 1991: 528) radica no

entre la ciencia que efectúa una construcción y la que no lo hace, sino entre la que lo hace sin saberlo y la que, sabiéndolo, se esfuerza por conocer y dominar lo más completamente posible sus actos, inevitables, de construcción y los efectos que, de manera igualmente inevitable, éstos producen.

Insistiendo en la naturaleza sociológica de la entrevista, Vallés (1997: 190) destaca «la consideración de la entrevista en tanto proceso de comunicación interpersonal, inscrito en un contexto social y cultural más amplio».

Así pues, la entrevista personal, semiestructurada y en profundidad es la técnica de producción de datos que mejor se adapta a la «esencia» (Mason 1996) de esta investigación, para conocer cómo el mundo simbólico orienta las prácticas de los sujetos dentro de los regímenes de género investigados y dar cuenta de sus procesos sociales de socialización/subjetivación y reproducción/cambio.

\section{Reflexividad y autorreflexión en las entrevistas: una oportunidad para repensar la metodología desde el compromiso social y ético con la investigación.}

No es frecuente en metodología plantear el trabajo de campo desde una perspectiva tan emic como lo voy a hacer yo aquí o, al menos, no lo es en so-

EMPIRIA. Revista de Metodología de Ciencias Sociales. N. ${ }^{\circ}$ 21, enero-junio, 2011, pp. 39-61 ISSN: 1139-5737 
ciología y, desde luego, resulta sintomático de que algo está cambiando en los planteamientos éticos, cualitativos y personales de la investigación social. Si utilizo estos términos, es porque de verdad creo que hay que referirse a la investigación, y a los sujetos con quienes se trabaja, en dichos términos.

Las entrevistas de la investigación tuvieron lugar en la última fase del trabajo de campo, tras el curso de educación afectivosexual realizado en el aula. Para ese momento, las chicas y chicos de clase y yo, investigadora y educadora simultáneamente, habíamos establecido una relación de complicidad centrada en la empatía, la confianza y la sinceridad. Mi actitud fue siempre abierta, respetuosa, cercana. Se generó una relación personal. Las sesiones de clase fueron, metodológicamente, un espacio preparatorio progresivo para hacer de la entrevista una conversación fluida, sincera, abierta, enriquecedora. Sin idealizarla. Como cabe esperar, no todas las personas se han sentido igualmente cómodas, siendo los chicos quienes más se cierran, tal vez por el hecho de ser yo mujer, tal vez por sus suspicacias, si bien, no ha sido así para todos. Para que la entrevista funcione, quien entrevista ha de ofrecer la misma actitud que espera de la persona a quien entrevista. Las entrevistas no son un episodio aislado en medio de las fases cronológicas de la investigación. De hecho, han sido parte integrada en el día a día de este trabajo. Han generado sus propias dinámicas, polémicas, intrigas y momentos llenos de sensibilidad y empatía. Están perfectamente integradas en todo el proceso de investigación acción educativa. No se trata de un hecho baladí. Pone de manifiesto la incidencia de la entrevista en la vida cotidiana del grupo de clase. Se saben elegidas/os para hacer este proyecto entre todos los grupos-clase del colegio. Intento buscar siempre un punto de equilibrio entre la cercanía con su tutor/a (como representante del profesorado del centro) y la cercanía con el estudiantado, del que me separan entre 10 y 13 años de edad, pero con el que comparto ciertas cosas como la música, la ropa, o algunos hobbies, todo lo que aprovecho para crear vínculos y referentes compartidos, para acercarme a ellas y ellos. Sé que no me ven como la profesora, ni como una investigadora ajena al centro. Me ven cercana, pero no tienen claramente definido mi rol en clase, como sí lo está el de su tutora o tutor. Confían en mí, porque les hablo de temas que les interesan mucho y lo hago sin ridiculizarles, ni ponerles en cuestión, ni hacerles juicios de valor; abiertamente, con madurez y sinceridad. En el momento de la entrevista, los primeros minutos son para ubicarse. Hay quien mira la grabadora, pero una vez que les explico que es para registrar bien la información y no olvidarme de nada, empiezan a hablar y, cuando se meten en la dinámica de la conversación, ya no se acuerdan de la grabadora, porque ahora lo que les interesa es hablar, de cosas que no suelen hablar con personas adultas, o de cosas que no pueden decir a su grupo de amistad, por la presión y el control que ejerce sobre cada miembro del grupo; a veces, reconocen que es la primera vez que se paran a reflexionar sobre algunas de las cuestiones que les voy planteando. La afectividad ha caracterizado la dinámica de las relaciones dentro y fuera del aula entre quien investigaba y quienes constituían el grupo de sujetos de la investigación. La valoración obtenida por el centro sobre esta experiencia 
fue siempre muy positiva, por la necesidad que destacaban de trabajar estos temas en clase y todo lo que estaban aprendiendo, y por lo bien que se sentían en clase.

Cuando se trabaja la dimensión ética de la investigación social, se plantea siempre la cuestión de la reciprocidad, pero la pregunta que más me inquietaba como investigadora era, ¿qué obtienen a cambio los sujetos que ceden trocitos de sus vidas para esta investigación? Se suele afirmar que la consideración, la visibilización, el reconocimiento de sus vidas, sus experiencias, sus palabras, suelen ser la gratificación más importante, más, incluso, que las retribuciones económicas, que mercantilizan el sentido original de la investigación social. Yo comparto plenamente esta idea, pero añado algo más. Esa misma gratificación procedente de la consideración y el reconocimiento la recibimos las personas que hacemos este tipo de investigación social. La cantidad de momentos entrañables justifica, con creces, la dimensión afectiva, ética y profesional de nuestro trabajo. Ése es el valor del trabajo docente, y de las relaciones sociales que se construyen a lo largo de un proceso de investigación social que comprende un período de tiempo más o menos amplio con las personas con las que se trabaja en la investigación. La clave es, sin duda, la reciprocidad. Por su temática, las entrevistas han sido, para los sujetos, un espacio para reflexionar, desahogarse, hablar y sacar fuera sentimientos, afectos, emociones o pensamientos no siempre fáciles de verbalizar. La investigación social puede, desde este otro punto de vista metodológico, convertirse en un espacio de reconocimiento (Skeggs 1997). Ésa es la palabra clave. Sin idealizaciones, pero desde el compromiso investigador, docente, ético y social.

\section{El protocolo de entrevista}

La entrevista ha sido el instrumento más importante de producción de datos en la metodología cualitativa de esta investigación, si bien, no ha sido el único. Como herramienta de investigación sociológica, la entrevista recoge las dimensiones propuestas en los objetivos de la investigación: valores, normas y prácticas sobre género, sexualidad, afectividad y cuerpo, referido todo ello a los regímenes de género del barrio, la familia, la escuela, el grupo de amistad y la pareja, con el punto de mira puesto en los procesos de socialización y subjetivación, para poder reparar en los procesos sociales de reproducción y cambio, atendiendo a las perspectivas comparativas de clase, género y etnia.

Para diseñar el protocolo, he tenido en cuenta, por un lado, la esencia de la investigación, así como los objetivos de la misma y, por otro, el estado de la cuestión, atendiendo especialmente a la obra de Connell $(1987,2002)$ y los principales trabajos realizados dentro de lo que podemos llamar sociología del amor (Beck y Beck-Gernsheim 2001; Giddens 2004; Gómez 2004; Bauman 2005; Bourdieu 2005; Castells y Subirats 2007), de donde he extraído los parámetros fundamentales que definen las cuatro estructuras de la política afectivo-

EMPIRIA. Revista de Metodología de Ciencias Sociales. N. ${ }^{\circ}$ 21, enero-junio, 2011, pp. 39-61 ISSN: 1139-5737 
sexual -género, sexualidad, afectividad y cuerpo-, a fin de fundamentar socioestructuralmente mi objeto de estudio (Venegas, 2009a).

Para la categorización de la información producida por las entrevistas he utilizado el programa informático de análisis cualitativo NUDIST. Los principales nudos han sido las cuatro estructuras sociales que componen la política afectivosexual. De estos nudos, parten árboles cuyas ramas se corresponden con las cuestiones de las entrevistas relativas a cada nudo. Este esquema de análisis utilizado para indexar las entrevistas con el NUDIST es el que he seguido posteriormente en la fase de análisis de las entrevistas.

Además de la entrevista, cabe mencionar también la observación participante durante el trascurso de las sesiones de clase en el curso de educación afectivosexual, y que ha producido datos de gran relevancia para complementar el análisis de las entrevistas. De este modo, toda la práctica investigadora se convierte en una práctica autorreflexiva que va adquiriendo sentido y significado en el transcurrir de la experiencia. Todos los sujetos que compartimos este espacio estamos aprendiendo algo. Ahí radica el mayor potencial de esta forma constructivista de llevar a cabo el proceso de enseñanza-aprendizaje como espacio de investigación.

Una tercera técnica de producción de datos, complementaria a las entrevistas, la constituyen las actividades individuales y grupales propuestas en cada sesión de clase. Estas actividades registran las temáticas trabajadas en el curso de educación afectivosexual y se complementan con el protocolo de entrevista, cuyo diseño parte de la revisión bibliográfica, dividida en dos bloques: los materiales sobre educación afectivosexual, como punto de partida de la investigación; y la teoría social feminista, para dar consistencia epistemológica y ontológica a la misma. En la teoría social feminista de las últimas décadas, el género es el enfoque para analizar y explicar las cuestiones relativas a la sexualidad, inseparable del cuerpo y vinculada -por tradición o para criticar esa tradición y dar cuenta de los cambiosal amor, la afectividad. Los trabajos feministas, aún cuando se centran en uno de estos temas, tienden a vincular los cuatro, porque están estrechamente relaciona$\operatorname{dos}^{15}$. En cuanto a los materiales sobre educación afectivosexual, han sido sometidos a un proceso de categorización hasta llegar a diseñar el programa desarrollado en clase, partiendo de la indización de todos los temas frecuentes o comunes a los materiales revisados (columna 2, tabla 2) y que, entiendo, se corresponden con cuatro categorías - género, sexualidad, afectividad y cuerpo (columna 1). Después, hube de reagrupar los temas para ajustarlos a las sesiones de clase cedidas por los colegios (columna 3), acompañando cada sesión de una actividad de clase (columna 4). La siguiente tabla recoge todo este proceso:

${ }^{15}$ En el capítulo de revisión bibliográfica de mi tesis doctoral (Venegas, 2009a) dedico amplio espacio a revisar la aportaciones de la teoría social feminista, buscando las relaciones entre ello a fin de definir mi objeto de estudio: la política afectivosexual, en un intento por aglutinar, en torno al mismo concepto, esas dimensiones estructurales relacionadas. Para ello, considero las cuatro categorías de la educación afectivosexual como las cuatro estructuras de relaciones que componen la política afectivosexual: relaciones de género, sexuales, afectivas y corporales. 
Tabla 2. Proceso de elaboración del programa de educación afectivosexual

\begin{tabular}{|c|c|c|c|}
\hline Categorías & Temas registrados & Reagrupación de temas & Actividad de clase \\
\hline \multirow{4}{*}{$\begin{array}{l}\text { 1. GÉNERO } \\
\text { (2 sesiones) }\end{array}$} & $\begin{array}{l}\text { 1.1. Valores éticos y so- } \\
\text { ciales de ciudadanía }\end{array}$ & \multirow{2}{*}{$\begin{array}{l}\text { 1.1. Estereotipos de gé- } \\
\text { nero. Sexo/género. } \\
\text { Desigualdad de género. }\end{array}$} & \multirow[t]{2}{*}{ Vamos a pintarnos. } \\
\hline & $\begin{array}{l}\text { 1.2. Teoría sexo-género. } \\
\text { Estereotipos de género }\end{array}$ & & \\
\hline & 1.3. Igualdad de género & \multirow{2}{*}{ 1.2. Violencia de género. } & \multirow{2}{*}{$\begin{array}{l}\text { Canciones de Melendi: } \\
\text { «Hasta que la muerte } \\
\text { nos separe», «El infor- } \\
\text { me del forense» }\end{array}$} \\
\hline & 1.4. Violencia de género & & \\
\hline \multirow{8}{*}{$\begin{array}{l}\text { 2. SEXUALIDAD } \\
\text { ( } 3 \text { sesiones incluyendo } \\
\text { la de Cruz Roja) }\end{array}$} & $\begin{array}{l}\text { 2.1. Diversidad cultural: } \\
\text { la construcción socio- } \\
\text { cultural de la sexualidad }\end{array}$ & \multirow{3}{*}{$\begin{array}{l}\text { 2.1. Sexualidad } \\
\text { Seres sexuados } \\
\text { Ciclo respuesta sexual }\end{array}$} & \multirow{3}{*}{ Ciclo respuesta sexual. } \\
\hline & $\begin{array}{l}\text { 2.2. Seres sexuados: de- } \\
\text { seo, atracción, actividad } \\
\text { sexual }\end{array}$ & & \\
\hline & $\begin{array}{l}\text { 2.3. Homofobia: diver- } \\
\text { sidad en la sexualidad }\end{array}$ & & \\
\hline & \begin{tabular}{|l|} 
2.4. Malos tratos y abu- \\
sos sexuales a la infancia
\end{tabular} & $\begin{array}{l}\text { 2.2. Diversidad de se- } \\
\text { xualidades }\end{array}$ & $\begin{array}{l}\text { Capítulo de Los Simp- } \\
\text { sons: «Los tres gays del } \\
\text { bloque» }\end{array}$ \\
\hline & $\begin{array}{l}\text { 2.5. Expresión de la se- } \\
\text { xualidad }\end{array}$ & \multicolumn{2}{|c|}{ Esta cuestión no pudo tratarse finalmente } \\
\hline & 2.6. Salud sexual, ETS & \multirow{2}{*}{\multicolumn{2}{|c|}{$\begin{array}{l}\text { Impartido por Cruz Roja (actividad programada } \\
\text { por el colegio) }\end{array}$}} \\
\hline & $\begin{array}{l}\text { 2.7. Prevención de em- } \\
\text { barazos no deseados }\end{array}$ & & \\
\hline & 2.8. Masturbación & \multicolumn{2}{|c|}{ Incluido en el ciclo de respuesta sexual } \\
\hline \multirow{7}{*}{$\begin{array}{l}\text { 3. AFECTIVIDAD } \\
\text { (2 sesiones) }\end{array}$} & $\begin{array}{l}\text { 3.1. Afectividad sana, } \\
\text { madura, responsable y } \\
\text { reflexiva }\end{array}$ & \multirow[t]{3}{*}{$\begin{array}{l}\text { 3.1. Expresión de senti- } \\
\text { mientos, enamoramien- } \\
\text { to, celos, confianza }\end{array}$} & \multirow[t]{3}{*}{$\begin{array}{l}\text { 3.1.Taller: «Las histo- } \\
\text { rias de amor» }\end{array}$} \\
\hline & 3.2. Respeto mutuo & & \\
\hline & $\begin{array}{l}\text { 3.3. Relaciones interper- } \\
\text { sonales y comunicación }\end{array}$ & & \\
\hline & $\begin{array}{l}\text { 3.4. Decir sí o no: toma } \\
\text { responsable de decisio- } \\
\text { nes en nuestras relacio- } \\
\text { nes sexuales }\end{array}$ & \multirow[t]{4}{*}{$\begin{array}{l}\text { 3.2. Relaciones interper- } \\
\text { sonales, afectividad, co- } \\
\text { municación, expresión } \\
\text { sentimientos }\end{array}$} & \multirow[t]{4}{*}{$\begin{array}{l}\text { 3.2. Taller: «La primera } \\
\text { vez» }\end{array}$} \\
\hline & $\begin{array}{l}\text { 3.5. Expresión de senti- } \\
\text { mientos }\end{array}$ & & \\
\hline & 3.6. Enamoramiento & & \\
\hline & 3.7. Celos y confianza & & \\
\hline \multirow{3}{*}{$\begin{array}{l}\text { 4. CUERPO } \\
\text { ( } 2 \text { sesiones) }\end{array}$} & $\begin{array}{l}\text { 4.1. Trastornos Alimen- } \\
\text { tarios }\end{array}$ & 4.1. Anorexia, bulimia & $\begin{array}{l}\text { Impartido por ADA- } \\
\text { NER (actividad progra- } \\
\text { mada por el colegio) }\end{array}$ \\
\hline & 4.2. Modelos belleza & 4.2. Modelos belleza & La historia de la belleza \\
\hline & $\begin{array}{l}\text { 4.3. Autoestima y aserti- } \\
\text { vidad }\end{array}$ & 4.3. Autoestima & $\begin{array}{l}\text { Fragmento de «Donde } \\
\text { el corazón te lleve» }{ }^{16}\end{array}$ \\
\hline
\end{tabular}

${ }^{16}$ Fuente: Tamaro, S. (1994). Donde el corazón te lleve. Madrid: Planeta.

EMPIRIA. Revista de Metodología de Ciencias Sociales. N. ${ }^{\circ}$ 21, enero-junio, 2011, pp. 39-61. ISSN: 1139-5737 
Además, se pasó un cuestionario de evaluación inicial y otro de evaluación final para medir si el curso había generado cambios entre el alumnado. Todas las sesiones de clase y las entrevistas fueron grabadas.

\section{ALGUNAS REFLEXIONES METODOLÓGICAS DESDE LA TEORÍA FEMINISTA}

Bajo el modelo de educación afectivosexual definido por los materiales revisados subyacen las críticas que la epistemología feminista dirige al patriarcado. Educar en esos temas (tabla 2) es formar sujetos en pro de la igualdad sexual y de género, pues es la materialización curricular de una crítica feminista que conlleva, igualmente, importantes reflexiones metodológicas, las cuales han permeado y guiado mi trabajo. En este apartado, me refiero a ellas por su relevancia en la metodología y el objeto de estudio que me ocupan.

\section{Sujeto, valores y emociones en la investigación social feminista}

Algunos principios esenciales del modelo actual de educación afectivosexual son aportaciones hechas por la metodología feminista al situar al sujeto -la persona- en el centro del trabajo; principios como la importancia de que quienes participan se sientan a gusto, se expresen en sus propios términos, vean respetada su integridad y reconocidas las diferencias y similitudes dentro del grupo (Szirom 1988; Barragán 1999; Gorrotxategui y de Haro 1999). Esta centralidad del sujeto es la base, también, de la apuesta feminista por reconocer la presencia de la subjetividad de quien investiga en su trabajo, como valor añadido, no como la debilidad científica que presupone el positivismo en ello (Nicholson 1990), ya que, según las críticas de marxismo, weberianismo y feminismo a la idea de objetividad defendida por el positivismo, «la objetividad (...) necesita dar cuenta de la subjetividad $\gg^{17}$ (Skeggs 1997: 33). Hay quien da incluso un paso más, proponiendo el término "respeto por la persona» ${ }^{18}$ (Reiss 2005), desde el que resulta clave focalizar la investigación en las significaciones que los sujetos hacen de su vida. Este principio metodológico guía también, como vimos, la propia entrevista, de manera que quien investiga ha de interpretar la situación de la entrevista según los valores y creencias que se construyen en esas situaciones de interacción (Jones 1985).

En investigación, no hay ni decisiones ni resultados exentos de valores pues, como afirma Jones (1985), la investigación requiere, por definición, una toma constante de decisiones que implican intereses de investigación y teorías

\footnotetext{
17 Traducción del original en inglés al español mía.

18 Traducción del original en inglés al español mía.
} 
previas. Pese a todo, el feminismo ha defendido el valor científico de esta forma de hacer y entender la investigación social, pues los criterios de cientificidad radican en la sistematización de teoría y metodología de manera que haya una conexión permanente entre las dimensiones ontológica, epistemológica y metodológica del proceso de investigación (Mason 1996), para que la producción de los datos sea adecuada (Skeggs 1997). De ahí que comenzara mi artículo especificando la «esencia de la investigación» (Mason 1996).

Estas reticencias positivistas se han dirigido también al reconocimiento de los afectos y emociones en la investigación social, al considerarlos un riesgo para la validez del conocimiento en Occidente (McLaughlin 2003). Por contra, desde la investigación acción ${ }^{19}$ se defienden por el estímulo que representan para el cambio social. Ignorar los sentimientos no resulta productivo en investigación, sobre todo cuando se trabaja en grupo (Abercrombie 1989; cf. McLaughlin 2003) o en un espacio altamente emocional como el de la enseñanza (Hargreaves 1994; Nias 1996; Noddings 1996; cf. McLaughlin 2003). A ello se suman otras dos razones sobre la importancia de las emociones en investigación acción educativa feminista, tal como plantea Burman (2006), por un lado, la implicación de las emociones en la cuestión de la participación; por otro, la consideración de las emociones como formas de acción en la investigación. Burman (2006) parte de dos supuestos: la 'emocionalización' de la vida pública y la entrada de ello en las prácticas educativas. Utiliza el término «geografías emocionales» para destacar la dimensión relacional de las emociones y afirma que la reflexividad, en tanto que práctica educativa e investigadora, configura el espacio para la participación.

\section{Ética, política, agencia y voz}

Es también la centralidad de las personas con las que se investiga lo que la lleva a Skeggs (1997) a considerar la metodología como una forma de teoría con consecuencias sociales y políticas, pues la investigación con personas tiene implicaciones sociales. La autora enfatiza, así, la dimensión ética que subyace a toda práctica investigadora, en la que considera capitales la autoridad y responsabilidad epistemológicas. Siguiendo a Foucault, Skeggs afirma que toda persona se ve envuelta en relaciones de conocimiento que producen, históricamente, relaciones de poder, y que legitiman las relaciones entre sujeto y objeto, algo que ha estado históricamente en manos de los hombres (género) blancos (etnia) de clase media (clase), mientras el resto de grupos sociales han sido otrizados, resultando un discurso científico normativo que evidencia cómo «las teorías soportan siempre la marca de sus marcadores $»^{20}$ (Skeggs 1997: 20).

${ }^{19}$ McLaughlin (2003) desarrolla ampliamente la relación ente investigación acción y emociones en el «Action Research in Teacher Education (ARTE) Project» que puede consultarse en http://www.educ.cam.ac.uk/arte/index.html.

${ }^{20}$ Traducción del original en inglés al español mía.

EMPIRIA. Revista de Metodología de Ciencias Sociales. N. ${ }^{\circ}$ 21, enero-junio, 2011, pp. 39-61 ISSN: 1139-5737 
Frente al desequilibrio de poder en el discurso científico positivista, esa apuesta del feminismo por la persona se refleja en la teorización de conceptos como empoderamiento y agencia (Heiskanenn 2006), de amplio desarrollo feminista desde los ' 80 en adelante y en relación a los que la investigación acción ha sido considerada como una metodología para el cambio, al incluir la idea de participación democrática en la acción y en la reflexión. Desde la perspectiva de género, añade Heiskanenn (2006), significa dar voz a quienes permanecen en silencio $^{21}$, las mujeres -a lo que cabe añadir los grupos históricamente subordinados-, para romper con las diferencias de poder. La autora recoge también la concepción generativa de la agencia de McNay (2000; cf. Heiskanen 2006), según la cual las personas enfrentan la complejidad de las situaciones cotidianas de forma innovadora, lo que incluye un potencial de cambio en que se vinculan las dimensiones material y simbólica del orden social ${ }^{22}$.

Mi trabajo había estado inspirado, en su concepción y diseño, por estas tesis feministas, de manera que he buscado siempre cuidar especialmente la dimensión ética implicada en todas las partes del proceso. Así, los dos colegios fueron informados detalladamente del proyecto antes de iniciar el trabajo de campo en ellos. Conocían el programa del curso sobre educación afectivosexual y que, seguidamente, iba a entrevistar a cada estudiante de la clase. Las puertas de la misma estuvieron siempre abiertas, y la tutora/tutor presente cuando así lo ha requerido, que ha sido en la práctica totalidad de las sesiones de clase. Las chicas y chicos de clase han sido protagonistas directos en todo el proceso. De hecho, uno de mis principales compromisos en este trabajo ha sido darles voz, escuchar y hacer oír esa voz, visibilizar sus demandas, para empezar a abrir espacio teórico y práctico en torno a la política afectivosexual en la adolescencia, pero con la intención de hacerlo desde la voz de los propios sujetos de la investigación (Venegas, 2007). Las chicas y chicos han estado permanentemente informados de lo que íbamos haciendo y de las razones de ello, bajo el objetivo de promover su capacidad reflexiva y crítica para favorecer la formación de un pensamiento autónomo, con criterio para tomar decisiones informadas.

En esta línea, resulta interesante el trabajo de Hadfield y Haw (2001) sobre la relación entre investigación acción, jóvenes y 'voz'. Los autores denuncian que, bajo la moda reciente de apelar al término 'voz' en el trabajo con jóvenes, subyacen prácticas de poder de quienes dan espacio a esa voz, especialmente las del profesorado cuando se investiga el terreno educativo. Para ellos, la gente joven es quien está en mejor posición para hablar sobre ser joven, pues puede hablar de su experiencia de una forma significativa; además, es necesario generar más espacios de diálogo entre profesionales y jóvenes; finalmente, la gente joven

${ }^{21}$ Esta misma idea es defendida también en mi trabajo (Venegas 2007).

${ }^{22}$ Destaco esta cuestión relativa a la agencia del sujeto porque es clave en relación al planteamiento epistemológico de mi trabajo, donde valores y normas -dimensión discursiva o simbólicaorientan las prácticas del sujeto, que tienen lugar en un contexto material en el que pueden contribuir a su reproducción o introducir espacios 
puede conseguir cambios en cuestiones que son de su interés si consigue que se escuchen sus voces. Pese a las buenas intenciones, la gente joven sigue encontrando importantes dificultades para participar activamente en las cuestiones sociales que le afectan, dada la tendencia social a silenciar su voz bajo el criterio de la minoría de edad o la falta de independencia socioeconómica. Mi trabajo, tanto en el aula como en las entrevistas, ha buscado ser un espacio real de reflexión, diálogo y debate donde visibilizar experiencias, opiniones y demandas, partiendo de una concepción del sujeto como agente de sus prácticas, con capacidad de influir sobre el entorno que le rodea, desde una investigación social sobre la política afectivosexual adolescente que pueda esclarecer cuestiones socialmente relevantes respecto a esta dimensión fundamental en la adolescencia. De ahí que fuera concebida como investigación social aplicada.

\section{CONCLUYENDO. UNA PROPUESTA METODOLÓGICA PARA EL CAMBIO SOCIAL}

El objetivo de este artículo ha sido recoger los pilares metodológicos de una propuesta de investigación sobre educación afectivosexual con el horizonte de contribuir a promover la igualdad sexual y de género, por lo que la opción por la investigación acción educativa emerge como metodología que mejor se ajusta a todo ello. Asimismo, otro de los puntos fundamentales del artículo ha sido mostrar cómo la metodología de investigación ha de ir imbricando de manera plausible todas las partes de la misma, a partir de su orientación epistemológica y ontológica, para asegurarse la calidad científica de la investigación, aún cuando se hace desde un posicionamiento teórico crítico con el positivismo clásico en investigación social. Para afianzar esta propuesta metodológica crítica y para el cambio social, he querido cerrar el artículo con algunas aportaciones fundamentales desde el feminismo que apuesta por una concepción metodológica alternativa donde subjetividad, afectividad, ética, valores y voz de las y los sujetos que se investigan ocupan un espacio central.

\section{BIBLIOGRAFÍA}

Barragán, F. (1999): Programa de Educación Afectivo Sexual. Educación Secundaria, Sevilla, Junta de Andalucía, Consejería de Educación y Ciencia e Instituto Andaluz de la Mujer.

Bauman, Z. (2005 [2003]): Amor líquido. Acerca de la fragilidad de los vínculos humanos, Madrid, Fondo de Cultura económica.

Beck, U. y BeCK-GERnSHeIM, E. (2001[1990]): El normal caos del amor, Barcelona, Paidós.

BERG, B. L. (2001 [1989]): Qualitative Research Methods for the Social Sciences, London, Allyn y Bacon.

Bourdieu, P. (coord.) (1999): La miseria del mundo, Madrid, Akal.

EMPIRIA. Revista de Metodología de Ciencias Sociales. N. ${ }^{\circ}$ 21, enero-junio, 2011, pp. 39-61 ISSN: 1139-5737 
-(2005[1998]): La dominación masculina, Barcelona, Anagrama.

BURMAN, E. (2006): «Emotions and reflexivity in feminised education action research», Educational Action Research, 14:3, pp. 315-332.

BUtLER, J. (2001): «La cuestión de la transformación social», en Mujeres y transformaciones sociales, Barcelona, El Roure, pp.7-30.

CARr, W. (1997): Calidad de la enseñanza e investigación-acción, Sevilla, Díada.

CASTElls, M. y SUBIRATS, M. (2007): Mujeres y hombres. ¿Un amor imposible?, Madrid, Alianza Editorial.

Connell, R. W. (1987): Gender and Power. Society, the Person and Sexual Politics, Stanford, Stanford University Press,

- (2002): Gender, Cambridge, Polito Press.

ElLIOT, J. (1993): El cambio educativo desde la investigación-acción, Madrid, Morata.

- (1994): La investigación-acción en educación, Madrid, Morata.

FERNÁNDEZ, F.; VENEGAS, M. y OLMEDO, A. (2004): «Fracaso escolar y exclusión social en la ciudad de Granada», en Exclusión / Inclusión social en la ciudad de Granada. Estudios y propuestas del Observatorio sobre Exclusión Social y Políticas de Inclusión (2003-2004): Síntesis, Granada, Maristán, pp.169-244.

Giddens, A. (1996): «Sociología». Madrid: Alianza, [2 $2^{\mathrm{a}}$ edición en español. Edición original en inglés, 1989].

GómEZ, J. (2004): El amor en la sociedad del riesgo. Una tentativa educativa, Barcelona, El Roure.

Gorrotxategui, M. y De Haro, I. M. (1999): Materiales Didácticos para la Prevención de la Violencia de Género. Unidad Didáctica para Educación Secundaria, Málaga, Consejería de Educación y Ciencia, Junta de Andalucía.

HADFIELD, M. y HAw, K. (2001): «Voice', young people and action research», Educational Action Research, 9:3, pp.485-502.

HEISKANEN, T. (2006): «Gender Issues in Action Research: Implications for Adult Education», International Journal of Lifelong Education, 25:5, pp.519-533.

Holland, D. C. y Eisenhart, M. A. (1990): Educated in Romance. Women, Achievement, and Collage Culture, Chicago, The University of Chicago Press.

Jones, S. (1985): «Depth Interviewing», en Applied Qualitative Research, Hants y Vermont, Gower, pp.45-55.

KeMmIS, S. (2007): «Action Research as a Practice-Changing Practice», en Investigaciónacción participativa. Reflexiones y experiencias, Segovia, E.U. Magisterio de Segovia y Collaborative Action-Research Network, disponible en http://hera.fed.uva.es/congreso/comunicaciones.htm [consulta: 22-6-2008].

Mason, J. (1996): Qualitative Researching, Londres, SAGE.

Mclaughlin, C. (2003): «The feeling of finding out: the role of emotions in research», Educational Action Research, 11:1, pp.65-78.

McrobBIE, A. (1978): «Working Class Girls and the Culture of Femininity», en Women's Studies Group: Women take issue, London, Hutchinson, pp.96-108.

Nicholson, L. J. (1990): «Introduction», en Feminism/Postmodernism, Londres, Routledge, pp.1-16.

ReIss, M. (2005): «Managing Endings in a Longitudinal Study: Respect for Persons», Research in Science Education, 35: pp.123-135.

SkEGGS, B. (1997): Formations of Class and Gender. Becoming Respectable, Londres, SAGE Publications.

STENHOUSE, L. (1987 [1981]): Investigación y desarrollo del curriculum, Madrid, Morata.

EMPIRIA. Revista de Metodología de Ciencias Sociales. N. ${ }^{\circ}$ 21, enero-junio, 2011, pp. 39-61. ISSN: $1139-5737$ 
SzIrom, T. (1988): Teaching Gender? Sex Education and Sexual Stereotypes, Londres, Allen \& Unwin.

TAYLOR, S.J. y BogdAN, R. (1986): «La entrevista en profundidad», en Introducción a los métodos cualitativos de investigación, Barcelona, Paidós, pp.100-132.

Urruzola, M. J. (1991): ¿Es posible coeducar en la actual escuela mixta? Una programación curricular de aula sobre las relaciones afectivas y sexuales, Bilbao, Maite Canal Editora.

VALLES, M. (1997): «Técnicas de conversación, narración (I): las entrevistas en profundidad», en Técnicas cualitativas de investigación social. Reflexión metodológica y práctica profesional, Madrid, Síntesis.

Venegas, M. y EChEVERría, C. (2005/06): «Social and Sexual-Affective Education at Secondary School. Implementing Gender Mainstreaming within Education for Peace and against Violence», International Journal of Learning, 12:7, pp.85-92. Disponible en http://www.Learning-Journal.com_[consulta: 14-10-2008]

VENEGAS, M. (2007): «Agencia y voz: Investigación acción en el contexto del proceso educativo», en Investigación-acción participativa. Reflexiones y experiencias, Segovia, E.U. Magisterio de Segovia y Collaborative Action-Research Network, en http://hera.fed.uva.es/congreso/ [consulta: 14-10-2008]

-(2009a): «La política afectivosexual: una aproximación sociológica a la educación afectivosexual», Granada, Universidad de Granada [Tesis doctoral publicada en formato digital].

-(2009b): «¿Cuál es el lugar de la sociología en la educación afectivosexual? La política afectivosexual», en La comunidad educativa ante los resultados escolares: XIII Conferencia de Sociología de la Educación Badajoz, 18 y 19 de septiembre de 2008, Barcelona, Milrazones.

WiLlis, P. (2003): «Foot Soldiers of Modernity: The Dialectics of Cultural Consumption and the $21^{\text {st }}$ Century School», Harvard Educational Review, 73:3.

\section{Webgrafía}

SEF (Sex Education Forum, integrado en National Children Bureau), en http://www.ncb.org.uk/Page.asp?originx_784wa_21042403840053g59p_200610203 $221 \mathrm{~g}$

SIECUS (Sexuality Information and Education Council of the United Status) en http://www.siecus.org/

\section{RESUMEN}

A veces, los trabajos de investigación en ciencias sociales forman parte de las propias prácticas transformadoras de quienes los realizan con el horizonte de promover, con ello, el cambio social. Este artículo versa sobre uno de esos casos. Durante la realización de mi tesis doctoral, sobre educación afectivosexual en dos colegios de la ciudad de Granada (España), y desde la mirada sociológica, el trabajo de investigación y el de acción educativa para el cambio social llegaron a confundirse hasta un punto tal que, casi sin querer, aunque muy conscientemente, pero, sobretodo, porque ésa era la demanda de la realidad social investigada, la investigación acción educativa devino la metodología de trabajo más in- 
dicada para investigar actuando en la educación para el cambio social hacia la igualdad sexual y de género. De todo este proceso metodológico, sistemático, respetando en todo momento el valor científico de la investigación, hablo en este artículo.

\title{
PALABRAS CLAVE
}

Investigación acción educativa, educación afectivosexual, política afectivosexual, cambio social.

\begin{abstract}
Sometimes, research works in social sciences join the transformative practices of the researchers themselves. In doing so, these researchers try to promote social change. This paper is about one of those works. During the realization of my $\mathrm{PhD}$ research, on sexual-affective education in two schools in the city of Granada (Spain), and from the sociological view, both the research, and the educational action work that looks for social change, converged to the point that, once we had taken account of the demand that the reality to be investigated was doing, educational action research became the most appropriate methodology in order to research acting on education for social change towards sexual and gender equality. In this paper, I will talk about all this process, which is methodological, systematic, and respectful with the scientific value of research.
\end{abstract}

\section{KEY WORDS}

Educational action research, sex and relationship education, sexual-affective politics, social change 